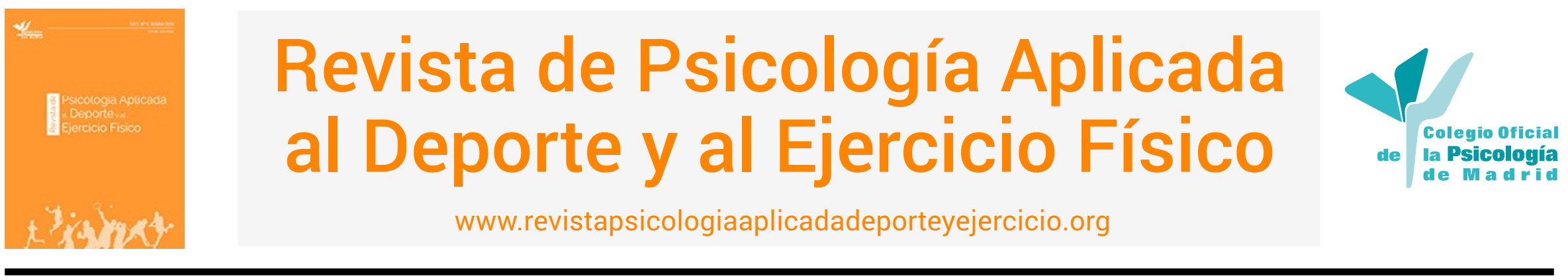

\title{
Psicología del jugador suplente en el fútbol
}

\section{Marcelo Roffé \\ Sociedad Latinoamericana y del Caribe de Psicología de la Actividad Física y del Deporte}

RESUMEN: El objetivo del presente trabajo es conocer en mayor profundidad sobre los jugadores de los equipos denominados "suplentes" y qué incidencia tiene esa condición a nivel psicológico y en su rendimiento deportivo. Para ello, se revisa esta cuestión, se presenta una experiencia profesional con un arquero de fútbol y se reflexionan las implicaciones prácticas desde la experiencia de 25 años del autor como psicólogo del deporte aplicado. Se observa que el estado de ánimo y el estrés del jugador suplente no es el mismo que el del jugador titular, y puede bajar su rendimiento ante esta situación. Existen ciertos factores psicosociales, emocionales y de preparación deportiva que colaboran para que pueda salir de ese puesto y lograr la titularidad. El compromiso al cambio y la mejora, la reflexión e introspección personal, tener buen diálogo con el entrenador para no tomarlo como algo personal y querer crecer, esforzándose cada día desde el entrenamiento de campo e invisible, serán la clave para poder superar ese estrés de no jugar y poder salir fortalecido. Se concluye que el jugador suplente a partir del apoyo personal y el entrenamiento psicológico puede mejorar su estado emocional, optimizar su preparación y rendimiento deportivo, así como lograr jugar con más asiduidad e incluso lograr la titularidad.

PALABRAS CLAVES: suplente, estrés, resiliencia, entrenador y autocritica.

\section{Psychology of the substitute player in soccer and other team sports}

ABSTRACT: The objective of this work is to gain more in-depth knowledge about the team players called "substitutes", and to explore the impact of this condition at psychological level and on their sports performance. To this effect, a professional experience with a soccer goalkeeper is presented, and the practical implications are reflected from the author's 25-year experience as an applied sports psychologist. It is seen that the state of mind and stress of the substitute player is not the same as that of the starting player, and his performance may decrease in this situation. There are certain psychosocial, emotional and sports preparation factors that can help the player to get out of that position and achieve tenure. Commitment to change and improvement, reflection and personal introspection, having a good dialogue with the coach so as not to take it personally and wanting to grow, striving every day from the field and invisible training, are the key to overcome the stress of not playing and be able to come out stronger. It is concluded that the substitute player, based on personal support and psychological training, can improve his emotional state, optimize his preparation and sports performance, as well as achieve more assiduous playing participation and even become a starting player.

KEYWORDS: substitute, stress, resilience, coach and self-criticism.

\section{Psicologia do jogador suplente no futebol e outros desportos coletivos}

RESUMO: 0 objetivo deste trabalho é conhecer em maior profundidade os jogadores das equipas denominados suplentes e qual o impacto dessa condição a nível psicológico e do desempenho desportivo. Para tal, esta questão é analisada através de uma experiência profissional com um guarda-redes, havendo também uma reflexão sobre as implicações práticas a partir da experiência de 25 anos do autor como psicólogo desportivo aplicado. Observa-se que o estado de espírito e stress do jogador suplente não são os mesmos do jogador titular, podendo o seu desempenho diminuir nesta situação. Existem certos fatores psicossociais, emocionais e de preparação desportiva que o ajudam a sair dessa posição e a alcançar a titularidade. 0 compromisso em mudar e na melhoria, reflexão e introspeção pessoal, ter um bom diálogo com o treinador para não ver a situação como uma crítica pessoal e ter vontade de crescer, esforçando-se todos os dias no treino de campo invisível, serão a chave para conseguir ultrapassar aquele stress de não jogar

Marcelo Roffé. Psicólogo Clínico y Deportivo. Presidente Sociedad Latinoamericana y del Caribe de Psicología de la Actividad Física y del Deporte (SOLCPAD). La correspondencia sobre este artículo debe enviarse al autor al e-mail: psicologia@marceloroffe.com 
e ser capaz de ficar mais forte. Conclui-se que o jogador suplente, com base no apoio pessoal e treino psicológico, pode melhorar o seu estado emocional, otimizar a sua preparação e desempenho desportivo, bem como conseguir um jogo mais assíduo e até mesmo tornar-se num jogador titular.

PALAVRAS-CHAVE: suplente, stress, resiliência, treinador e autocrítica.

Artículo recibido: 01/10/2020 | Artículo aceptado: 16/11/2020

La Psicología del Deporte es una especialidad consolidada de la Psicología, que busca estudiar y dar respuesta a los diferentes acontecimientos y demandas que se dan dentro del contexto deportivo (Cantón, 2016; COP, 1998; García-Naveira, 2010), en la cual el rol profesional del psicólogo/a del deporte (CGPE, 2020), se ha asentado en el fútbol en los últimos años (García-Naveira, 2018; Morelló et al., 2018; Roffé, 2016).

Un caso particular en el fútbol, y otros deportes de equipo, es la situación deportiva de "ser jugador suplente", una experiencia muy interesante de análisis y determinante en los equipos a lo largo de una temporada. Además, se entiende que "estar en la banca" o "ir al banco de suplentes", es un tema que afecta a todos/as los que forman parte de los equipos interdisciplinares, que trabajan en el apasionante mundo del alto rendimiento deportivo. Por ello es que este artículo espera abrir un nuevo espacio para pensar sobre el rol del psicólogo/a del deporte en relación a los jugadores/ as suplentes.

Para ello, en primer lugar, se valorarán ciertos estudios sobre esta temática, como referente del estado psicológico de dicha situación, y posteriormente, se presenta una experiencia profesional con un futbolista de elite, que inició como suplente, y tras el trabajo psicológico, logró ser titular. Además, se recorrerán ideas sobre esta cuestión que se dan como resultado de los 25 años de experiencia profesional del autor como psicólogo aplicado al deporte.

Partiendo de la Real Academia Española (RAE, 2020), etimológicamente este vocablo viene del antiguo participio del verbo activo transitivo «suplir» y del sufijo «-nte», que indica que hace la acción, del adjetivo que suple a una persona. Esto es, el jugador no juega de titular en el equipo y se encuentra en el banquillo, esperando su oportunidad para suplir a un compañero, decisión tomada por el entrenador en algún momento del partido.

A nivel comportamental (cognición, emoción y conducta), diferentes trabajos (De la Vega et al., 2011; Goire, 2010; Mateo, 2020; Olmedilla et al., 2011; Peris-Del Campo, 2019; VaIle-Chauvet et al., 2010) vinculan el jugador suplente con una "situación peor" frente al jugador titular, como, por ejemplo, mayor ansiedad, estado de ánimo negativo (e.g., hostilidad y depresión), baja confianza y variación en la situación dentro del equipo (e.g., cooperación y cohesión), menor rendimiento, descenso de la preparación deportiva, bienestar y cuidado fuera de la parcela deportiva (e.g., alimentación y descanso).

Por lo general, el jugador suplente quiere ser titular, sin embargo, no existe mucho material respecto de las características que debe tener un buen suplente ni sobre lo que se espera de él o de ella como suplente. También hay que tener presentes las diferencias entre deportes y los tiempos de los mismos, que afectará a la mayor o menor participación del suplente, por ejemplo, en balonmano, baloncesto y béisbol, entro otros deportes, donde se sabe que la rotación es alta, a diferencia de deportes como el fútbol, donde la rotación no es tan común o continua como en los deportes antes mencionados.

Los jugadores titulares están significativamente más satisfechos con la frecuencia de su participación con el equipo, su estado en general, rendimiento y resultados, que los jugadores suplentes. Tienen una relación mucho más estrecha y positiva con el entrenador y un concepto más positivo de sus propias aptitudes y perspectivas que los suplentes. También, los jugadores suplentes suelen percibir que los titulares están más apoyados y valorados por otras personas en relación al rendimiento y actitudes de trabajo, por ejemplo, por parte del entrenador. Al respecto, los jugadores suplentes suelen criticar más al entrenador de una manera más categórica en lo que respecta a la evaluación de rendimiento tendenciosa y a la organización inadecuada del equipo, que los jugadores titulares.

Considerando estas cuestiones, parece necesario conseguir que los entrenadores sean más sensibles a los procesos dinámicos de sus equipos, al objeto de reconocer los deportistas con problemas tan pronto como sea posible, anticipar y conducir adecuadamente los conflictos (Buceta, 2004). Esto también significa hacer que ellos sean más conscientes de su clase de mando y de su efecto sobre los jugadores individuales y sobre todo el equipo.

Un buen entrenador/líder es el que tiene motivados a los suplentes (Roffé y Rivas, 2013). No hay duda de que en cualquier deporte de equipo en el que se hable, el jugador/a suplente debería ser una solución y no un problema durante 
una temporada regular (e.g., 9 meses), en el que existen múltiples factores que pueden "imponer" la necesidad de participación del jugador suplente (e.g., expulsiones, lesiones, etc.).

A nivel general, en el fútbol profesional, se sabe que las intervenciones psicológicas dentro de los equipos se centran principalmente en formación, asesoramiento e intervención psicológica con el cuerpo técnico, entrenamiento psicológico con jugadores y equipo, entrenamiento psicológico integrado en las propias sesiones de preparación, intervención psicológica durante la competición y concentración de partido o competición, selección de jugadores, intervención psicológica con los servicios médicos y lesiones deportivas, el retiro del jugador, entre otros (García-Naveira, 2018). Y en concreto, el establecimiento de objetivos, el desarrollo de la cohesión, fortalecimiento de los aspectos mentales, en tanto manejo de las presiones, focalización de la atención y desarrollo de la autoconfianza, etc. (Roffé, 2016).

Además, el entrenamiento psicológico con un jugador de fútbol suplente para optimizar sus recursos psicológicos (e.g., regulación emocional, establecimiento de objetivos y mejora de la confianza) y de preparación (e.g., rendimiento en los entrenamientos y cuidados fuera de los mismos), es un tema abordado dentro de la Psicología del Deporte, cuyo objetivo perseguido es llegar a ser titular (Peris-Del Campo, 2019).

Partiendo de todas estas cuestiones, a continuación, se presenta una experiencia profesional con un jugador de fútbol suplente junto a unas reflexiones personales.

\section{Experiencia Personal}

Breve descripción del caso: arquero de 24 años, suplente en su actual equipo (primera división del fútbol argentino), el cual salió de su club original donde era suplente hacía mucho tiempo de un arquero de mucha trayectoria y de selección nacional, para ir a un club menor (actual). Llega a la demanda del consultorio pensando en que tomó una mala decisión porque en este club también era suplente, y poniendo en factores externos la causa de su suplencia.

El objetivo del trabajo fue fortalecer la autoconfianza, despejar su negativismo y miedos, aumentar la motivación, y que lograra centrarse en su esfuerzo para la búsqueda de la titularidad, entendiendo que en su rol inicial de suplente realizaba un aporte importante para el equipo. Finalmente logró la titularidad y jugar 13 partidos consecutivos en un buen nivel (más que en toda su carrera, hasta que llegó la pandemia del COVID-19 y la interrupción deportiva).

Se realizaron un total de 16 sesiones de 50 minutos cada una, con una frecuencia de una vez a la semana, en un consultorio privado, en formato presencial. Se aplicó una metodología de evaluación-intervención de orientación cognitivo-conductual (Ezquerro, 2015). Se realizaron entrevistas (semi-estructuradas), se aplicaron test, cuestionarios (CPRD-F, POMS, inventario miedos y presiones, test de Wartegg, test de persona bajo la lluvia, etc.) y se crearon auto registros (Roffé, 2009). Además, se realizó un entrenamiento en relajación (control de la respiración), práctica imaginada, establecimiento de metas, auto dialogo positivo y reestructuración cognitiva (Ezquerro, 2015). Como complemento, se utilizaron videos propios y ajenos y películas como medio para reflexionar y aprender y aplicaciones del móvil para controlar la ansiedad y mejorar la concentración. A continuación, se presenta parte del testimonio del arquero sobre su situación de suplente, el cual se irá valorando a lo largo de este apartado.

Ser arquero es el puesto más difícil del fútbol, ya que en los demás puestos si te destacas en los entrenamientos, unos minutos te vas ganando un lugar para competir, pero en este puesto todo es más difícil.

Arquero: "Lo de la cara larga pasa mucho. Cuando sos suplente te sentís fuera del equipo y del plantel. Te sentís que sos inferior al que juega. Y sentís que el técnico tiene algo en contra tuyo y no es así. El técnico tiene otra opinión que no te pone, pero vos lo sentís personal. Lo de la cara larga pasa mucho y si te queres hacer el rebelde te sale todo mal."

Acá podemos subrayar la importancia de salir de esa frase tramposa "no pido explicaciones cuando me pone, tampoco cuando me saca" y poder dialogar con el entrenador y preguntarle ¿qué me falta? Y no, ¿por qué no juego?, y así eliminar los fantasmas personales que poco ayudan a crecer.

Arquero: "Tenes muchos técnicos que a los que no jugaron el fin de semana ni tuvieron participación, les toca hacer fútbol un día después del partido y el entrenador no está y no te mira y vos te sentís desmotivado! Estas esperando que la cabeza del grupo, venga te mire y vea que estas bien, y ves al ayudante de campo y no es lo mismo a que este el principal y eso te desmotiva mucho también, el mensaje es muy duro".

El ejemplo de eso es Alfio Basile, gran entrenador, pero todo el tiempo declaraba "quiero recitar los once de memoria". Esa frase desmotiva al resto que no juega. Y el contraejemplo es Carlos Bianchi en Boca. Llegaba de jugar Copa Libertadores a la madrugada y al otro día sin dormir iba a ver la práctica de los que no viajaron. Y los jugadores siempre se lo destacaron.

Arquero: "A mi arrancar jugando y después no jugar nunca me pasó. He tenido compañeros que eran titulares y después suplentes y le han pasado muchos de estos puntos que vos citas" (ver más adelante consecuencias de la pérdida de la titularidad). 
"Nunca fui tercer arquero, pero veo el trabajo del tercer arquero y es muy bravo. Poca y nada de participación. A veces ni haces fútbol. Principalmente son los chicos más jóvenes, pero es muy duro."

Por algo Guardiola en su video motivacional pre-partido antes de un match clave vs. Manchester en Barcelona en el año 2009, comienza el video enfocando al tercer arquero. Todo dicho. El no reconocido.

Arquero: "Puede llegar a ser común que algunos jugadores que no juegan quieran que el entrenador pierda así dimite. No es mi caso porque yo siempre traté de ser lo más positivo, pero por hablar con compañeros o durante el partido en el banco de suplentes hay compañeros que quieren que le vaya mal al dt o especificamente a un jugador, es totalmente real. No es hacerle la cama al dt, pero creen que pueden cambiar la ecuación si ingresan".

Esto fue a raíz de Ricardo La Volpe que dijo públicamente que en Boca había suplentes que deseaban que le fuera mal.

Arquero: "El arquero es una posición que solo ataja uno y cuando el titular tiene un buen rendimiento puede estar mucho tiempo en el arco, años, y vos tenes que ser suplente y tenes que esperar tu momento y a veces se hace muy difícil, pero yo lo he trabajado con vos y es un estado mental de cómo estás en el momento. Porque por ahí vos estás en tu mejor momento el suplente, te sentís muy bien para jugar y no te toca, y por ahí se lesiona el arquero titular y vos no estás al cien, te toca jugar y lo haces mal, y es la única oportunidad que tenías en ese club".

El puesto también condiciona tu estado emocional. Y es muy difícil trabajar solo la construcción de la paciencia y el manejo de la ansiedad. Con ayuda de un psicólogo/a se facilita el proceso.

Los subtemas que hemos desarrollado y se quiere dejar planteados, luego de tantos años en el campo aplicado como psicólogo son:

- Motivación y desmotivación del suplente.

- El estado de ánimo del titular y del suplente (emociones positivas y negativas).

- El estrés del titular.

- Características del suplente negativo y del suplente positivo.

- La importancia del elogio del entrenador.

- Porque un buen entrenador líder es el que sabe comunicar.

- El desafío del entrenador en el plano motivacional de lograr que todos sus conducidos estén motivados.

- El concepto de suplente como solución.

- Son los jugadores con su rendimiento los que se ponen y se sacan.

Como indican Weinberg y Gould (2010), el elogio brinda un reconocimiento positivo y ayuda a los deportistas a con- tinuar su esfuerzo por mejorar, en especial a los que tienen poco reconocimiento como los deportistas suplentes.

$Y$ entender que la pérdida de la titularidad en muchos casos es igual a:

- Estrés

- Conflicto con compañeros

- Conflicto con el entrenador

- Conflicto con los dirigentes

- Pérdida de autoconfianza

- Disminución de autoestima

- Exceso de agresión

- Déficit atencional

- Déficit motivacional

- Sensación de frustración o fracaso

Es importante destacar lo que nos dicen Weinberg y Gould (2010) respecto de la comunicación entrenador-deportista y los cuidados que hay que tener con los fallos. El entrenador le dice a un deportista que si continúa trabajando bien en las prácticas estará entre los titulares cuando comience la temporada. A lo largo de las semanas subsiguientes, el entrenador halaga al deportista con frecuencia y no dice nada acerca de que no será titular. Dos días antes del comienzo de la temporada el deportista recibe un golpe cuando se ve en la lista de los suplentes. En este caso el entrenador debería haber sido más específico acerca del criterio para ser titular y debería haberle dado al deportista reportes contínuos.

Una jugadora de handball que he asistido hace un tiempo (jugaba en la liga francesa), le preguntó a la entrenadora que le faltaba y esta le señaló que no veía en ella compromiso en la marca en el retroceso. Eso le generó bronca ya que consideraba que en ataque hacía la diferencia, pero luego de asimilarlo, lo pudo modificar y logró ingresar muchos mas minutos que antes por partido.

Roberto Perfumo, ex futbolista mundialista argentino y ex entrenador en su libro "Jugar al fútbol" (1998) decía que a veces el jugador necesita sentir el frío del banco de suplentes en el trasero. También hay entrenadores que dan más lugar, minutos y cambios a los suplentes y los que menos (más conservadores). Es otro elemento a tener en cuenta de ese perfil de entrenador cuando el deportista debe elegir a donde ir. No está de más decir que el segundo gol más importante de la historia del prestigioso club argentino River Plate, lo realizó el futbolista Juan Fernando Quintero, colombiano que saltó desde el banco de suplentes por orden de Marcelo Gallardo y ajustició a Boca Juniors en la famosa final de la Copa Libertadores del 2018 realizada en el mismísimo estadio Santiago Bernabéu.

Aportes de otros futbolistas:

Jorge Valdano a Iván Zamorano: "si tengo 5 delanteros, vos sos el sexto". Me voy a quedar y voy a ser pichichi res- 
pondió el número nueve. Y así fue (Mateo y Valdano, 1999).

Finalmente, decir que se pusieron muchos ejemplos muy conocidos mundialmente de suplentes "exitosos" como Ivan Zamorano, Juan Fernando Quintero, Haaland, Lewandosky, etc. $Y$ de entrenadores en intervenciones positivas y negativas como Bianchi, Pekerman, Gallardo, La Volpe, etc.

Por último decir que José Pekerman en el Mundial de Brasil 2014 (Roffé, 2016), como Colombia ya estaba clasificada para octavos de final tras haber ganado los dos primeros partidos, frente a Japón, puso nueve suplentes siguiendo el ejemplo de Phil Jackson, gran líder de la NBA, quien en su libro "Canastas Sagradas" (Jackson y Delehanty, 2007) refiere que a veces prefería arriesgar un resultado apostando a la cohesión grupal, ya que sabía que a la larga ese fenómeno de la cohesión grupal iba a brindar muchos más resultados que el de un solo partido. En síntesis, no prometer, hacer que todos jueguen. Luego de ese partido Colombia había hecho jugar 22 de 23 jugadores, venció a Japón 4 a 1 y luego eliminó a Uruguay 2 a 0 en octavos de final, en su triunfo más importante en un Mundial de Fútbol.

\section{Discusión}

Es central trabajar con los y las deportistas suplentes de equipos, ya sean de deportes con alta o baja rotación. Clarificar el rol de estos deportistas en tanto suplentes, así como pensar el papel fundamental que cumple el entrenador como líder en la percepción de este rol del ser suplente.

Es pertinente indagar sobre el estado anímico de los suplentes que permitan desarrollar estrategias específicas para su aceptación y no funcionen como relegados, o bien como un castigo dentro del equipo.

Desde la Psicología Aplicada al Deporte, todavía hay mucho para desarrollar en relación a este tema. Para el futuro sería conveniente realizar nuevas investigaciones que permitan diferenciar y arrojar más luz sobre el rol del jugador suplente, sus implicancias y emociones.

A modo de conclusiones del trabajo:

- La Psicología del Deporte aporta herramientas para los deportistas con autocritica

- Si "el suplente" quiere crecer será una actitud y motivación y no un estado.

- No es beneficiosa la dicotomía "ganadores o perdedores" / "titulares o suplentes" ya que los límites se pueden borrar fácilmente.

- Los entrenadores deben incorporar herramientas psicológicas para ser más asertivos y comunicar mejor.

- Construir "equipos competitivos" es tener dos o más por puesto con posibilidades de ser titulares.
- El desafío profesional del psicólogo/a es ayudar a los suplentes para que un día sean titulares, reinterpretando la situación, aprendiendo a manejar el estrés y construyendo/fortaleciendo la resiliencia, brindándoles las herramientas psicológicas necesarias.

\section{Referencias}

Buceta (2004). Estrategias Psicológicas para entrenadores de deportistas jóvenes. Editorial Dykinson.

Cantón, E. (2016). La especialidad profesional en Psicología del Deporte. Revista de Psicología aplicada al Deporte y al Ejercicio Físico, 1(1), Artículo e2. http://doi.org/10.5093/rpadef2016a2

Colegio Oficial de Psicólogos de España (COP, 1998). Perfiles profesionales del psicólogo. Autor. https://www.cop.es/perfiles/ contenido/deporte.htm

Consejo General de la Psicología de España (CGPE, 2020). Acreditación nacional del psicólogo experto en psicología del deporte. http://www.acreditaciones.cop.es/

De la Vega, R., Ruiz, R., García G. y Del Valle, S. (2011). El estado de ánimo precompetitivo en un equipo de fútbol profesional: un estudio entre jugadores titulares y suplentes. Cuadernos de Psicología del Deporte, 17(2) 107-117.

Ezquerro, M. (2015). Evaluación conductual e intervención psicológica en el deporte, En A. García-Naveira y L. Locatelli, (Eds.), Avances en Psicología del Deporte (pp. 3-33). Paidotribo.

García-Naveira, A. (2010). El psicólogo del deporte en el alto rendimiento: aportaciones y retos futuros. Papeles del Psicólogo, 37(3), 259-268

García-Naveira, A. (2018). Pasado, presente y futuro del psicólogo del deporte en el fútbol español. Revista de Psicología aplicada al Deporte y al Ejercicio Físico, 3(1), Artículo e2. https://doi. org/10.5093/rpadef2018a8

Goire, L. (2010). Consideraciones acerca de los jugadores de reemplazo en el béisbol. Efdeportes, 15(144). https://www.efdeportes.com/efd144/los-jugadores-de-reemplazo-en-el-beisbol. $\underline{\text { htm }}$

Jackson, P. y Delehanty, H. (2007). Canastas Sagradas. Paidotribo.

Mateo, M. (2020). Cooperación y cohesión deportiva en deportistas amateurs de San Antonio de Areco. Trabajo de fin de Licenciatura. Facultad de ciencias jurídicas y sociales, Universidad Fundación UADE.

Mateo, J. y Valdano, J. (1999). Liderazgo. Grupo Santillana de Ediciones.

Morelló, E., Bert, B. y Navarro, S. (2018). Establecimiento de objetivos en el curriculum formativo de los futbolistas. Revista de Psicología aplicada al Deporte y al Ejercicio Físico, 3(1), Artículo e3. https://doi.org/10.5093/rpadef2018a7

Olmedilla, A., Ortega, E., Almeida, P., Lameiras, J., Villalonga, T., Sousa, C., Torregrosa M., Cruz J. y García-Mas A. (2011). Cohesión y cooperación en equipos deportivos. Anales de Psicología, 27(1), 232-238

Peris-Delcampo, D. (2019). Intervención Psicológica a distancia con un futbolista profesional: estudio de caso. Revista de Psi- 
cología Aplicada al Deporte y al Ejercicio Físico, 4(2), Artículo e15. https://doi.org/10.5093/rpadef2019a14

Perfumo, R (1998). Jugar al fútbol. Editorial Perfil.

Real Academia Española (RAE, 2020). Suplente. https://dle.rae.es/ suplente

Roffé, M. (2016). La preparación psicológica de la Selección Nacional Absoluta de Colombia para el Mundial de Fútbol Brasil 2014. Revista de Psicología Aplicada al Deporte y al Ejercicio Físico, 1(1), Artículo e3. https://doi.org/10.5093/rpadef2016a3
Roffé, M. y Rivas, C. (2013). El partido mental en 400 frases y 45 temas. Lugar editorial.

Roffé, M. (2009). Evaluación psicodeportológica: 30 tests psicométricos y proyectivos. Lugar editorial.

Valle-Chauvet, C. y Hernández-Pozo, M. (2010). Efecto de una intervención psicológica breve sobre la ejecución futbolística profesional. Revista Mexicana de Análisis de la Conducta, 36(1), 99-116.

Weinberg, R. S. y Gould, D. (2010). Fundamentos de la Psicología del deporte y del ejercicio físico. Editorial Médica Panamericana. 